\title{
Desarrollo del Pensamiento Crítico a partir de una estrategia pedagógica fundamentada en los Estándares Intelectuales aplicada en filosofía para los estudiantes de 11 A del Instituto Técnico Padre Manuel Briceño Jáuregui Fe y Alegría
}

Héctor Julián Machuca Ortiz ${ }^{1}$

Recibido: 23-04-2018

Aceptado: 20-05-2018

\section{RESUMEN}

Este artículo de investigación, abarcó el análisis de una propuesta pedagógica fundamentada en los estándares intelectuales de Paul Richard y Linda Elder enfocada al desarrollo del pensamiento crítico en filosofía para los estudiantes del grado undécimo A del Instituto Técnico Padre Manuel Briceño Jáuregui Fe y Alegría. Por ende, se diseñaron y aplicaron estrategias que fortalecieron los estándares de claridad, certeza, relevancia, lógica, justicia, precisión, profundidad y amplitud en la Propuesta Pedagógica, articulándose con los elementos de pensamiento y virtudes intelectuales que, además, permiten la formación de buenos pensadores críticos. Sin embargo, desde el análisis y valoración de los resultados más significativos, se debe mencionar que los estudiantes no desarrollaron el pensamiento crítico como se había estructurado, debido a esta dificultad se diseñó unas rejillas que orientaron adecuadamente la construcción de preguntas de selección múltiple y estrategias de aula que fortalecen las habilidades de los educandos en los estándares intelectuales y elementos del pensamiento.

Palabras clave: Pensamiento Crítico, Estándares Intelectuales, Elementos Del Pensamiento, Virtudes Intelectuales, Propuesta Pedagógica.

1. Universidad Autónoma de Bucaramanga. Facultad De Ciencias Sociales Humanidades y Artes

Correo: hmachuca@unab.edu.co-30deenerode1985@gmail.com ORCID: https://orcid.org/0000-0003-4079-2180 


\title{
Development of Critical Thinking based on a pedagogical strategy based on the Intellectual Standards applied in philosophy for the $11 \mathrm{~A}$ students of the Padre Manuel Briceño Jáuregui Technical Institute Fe y Alegría
}

\begin{abstract}
This article of research, is an analysis of a pedagogic proposal to eleventh A grade students in Instituto Técnico Padre Manuel Briceño Jáuregui Fe y Alegría. This proposal tends to focus on the development on critical thinking in philosophy subject taking into account the intellectual standards proposed by Paul Richard and Linda Elder. For That reason, it was designed and applied for strategies which strengthened standards of clarity, accuracy, precision, consistency, relevance, good reasons, depth, breadth, and fairness in this pedagogical proposal. Therefore, these were articulated with the critical thinking elements and intellectual values, which all together may give as a result correctness process to educate excellent thinkers. However, after analyzing and assessing the relevant outcomes obtained in this study; the research shows that the students have not developed critical thinking as it was though. So, thanks to this trouble, it was designed a grille which have been helping students; not only, to understand how to write multiples chose questions, but also classroom strategies. In this way, these aspects support to increase students' abilities, intellectual standards and thinking elements.
\end{abstract}

Keywords: Critical Thinking, Intellectual Standards, Elements Of Thought, Intellectual Virtues, Pedagogical Proposal .

\section{Introducción}

Existe en nuestra actualidad la urgencia de promover el desarrollo de habilidades cognitivas los estudiantes, debido a que vivimos en un entorno de avances acelerados de la tecnología y que le acompaña la gran masificación de información por todos los medios de comunicación sin criterios de certeza o validez, lo que nos lleva a creer que todo es cierto, 
sin la mínima capacidad de discernir entre la verdad y la falsedad. Por otro lado, falta la rigurosidad en la valoración de argumentos, analizar las fuentes, comprender si se está basando en prejuicios o supuestos para validar unos datos que se presentan como verdaderos (Aparicio, 2018).

Concretando este fenómeno en la práctica del docente, se identifica diferentes habilidades del pensamiento que deben ser desarrolladas en el aula, como son: comprender, validar, analizar, valorar, discutir e inferir la verdadera intención del autor, identificar los datos implícitos y escrudiñar lo que permanece oculto (Pérez, 2013). Para ello es necesario fortalecer el desarrollo de las habilidades de análisis de información en las diferentes asignaturas a través del pensamiento crítico, pero ante todo, que conlleve al estudiante a ser el protagonista de su propio aprendizaje (Viejo, Cabezas, martínez, 2013), que comprenda lo que está haciendo y sea capaz de generar un aprendizaje significativo, es decir, que desarrolle su pensamiento.

Así mismo, el educando mediante la aplicación de una propuesta pedagógica del fortalecimiento del pensamiento crítico mejorará sus habilidades en interpretar, analizar, validar la información para no ser tomada a la ligera (de la Calle et al., 2014), transformando su forma de pensar en pro de la resolución de problemas de la vida personal y en la hora de tomar de decisiones. Por otro lado, implementará la actitud filosófica de la duda, la pregunta, la sospecha frente a la información presentada en las clases, analizando si la información recibida y el conocimiento construido permiten transformar la realidad social de su propio contexto. Como lo relaciona Fernández (2006), "el educando debe aprender a buscar, identificar, analizar y valorar la información para construir el conocimiento; además, se debe propiciar la reflexión de lo que se está haciendo, el cómo lo está haciendo y los resultados obtenidos para mejorar su propio desempeño y aprender de forma crítica" (p. 4243).

\section{Referentes teóricos}

El desarrollo del pensamiento crítico a través de una propuesta pedagógica basada en los estándares intelectuales se enfoca en tres líneas teóricas que brindan luces al trabajo en el aula, realizándose en torno a la validación de la información o de textos para la construcción del conocimiento. 
Para enfatizar, las tres líneas teóricasson: Primero, la de Linda Elder y Paul Richard de los cuales se retoma su propuesta de Estándares Universales, Elementos del Pensamiento y Virtudes Intelectuales. Segundo, desde la teoría de Matthew Lipman, gracias a su propuesta de la filosofía para niños en la que realiza una estrategia que permite desarrollar las virtudes intelectuales que son las comunidades de indagación y el plan de discusión. Y, como tercera línea, la teoría del pensamiento crítico de Facione o del argumento como camino al pensamiento crítico, estructura una metodología que complementa la propuesta de los estándares universales.

\section{Definición del pensamiento crítico}

Para Paul y Elder (2005), "el pensamiento crítico es un proceso de analizar y evaluar el pensamiento con el propósito de mejorarlo, debido a que se apoderan de sus estructuras inherentes del acto de pensar y por someterlas a los estándares intelectuales" (p. 7).

\section{A. Linda Elder y Paul Richard}

\section{*Estándares Intelectuales}

Elder (2005) afirma: "Los Estándares Intelectuales (E.I.) ayudan a pensar mejor" (p. 10), es decir, permiten evaluar el pensamiento para mejorarlo.

Claridad: Se refiere a la habilidad de comprender lo que se lee, el sentido de la lectura. Para evaluar este estándar se plantea una pregunta sencilla ¿Cuál es el tema del texto? Además, las estrategias para desarrollar este estándar son parafrasear y dar ejemplos.

Certeza: Este estándar evalúa el pensamiento en su capacidad de comprobar la validez de una información. Para ello es necesario que los estudiantes planteen unos criterios de análisis y los apliquen a la información para comprobarla. Se inicia con preguntas como "¿Estás seguro de que es verdad? ¿Cómo verificar si algo es verdadero (Criterios)? ¿Cómo verificar si esta información es cierta o falsa?" (Elder, 2005, p. 12).

Relevancia: Se debe verificar la importancia de las consideraciones frente al problema del texto, es decir si el autor está usando información relevante para abordar el problema tratado. Las preguntas que se pueden abordar son las siguientes: “¿Cuál es la idea central del texto? ¿Cómo se 
relaciona esta información con el tema del texto? ¿Qué información es útil para responder esta pregunta?" (Elder, 2005, p. 13).

Lógica: Para evaluar el pensamiento, es necesario analizar, descomponer el texto en sus partes y ver cómo se combinan entre sí. Para ello, se debe interrogar sobre ¿Cuál es la estructura del texto? ¿Cómo encaja este dato particular en la estructura general? ¿Cuál es el párrafo que sigue, teniendo en cuenta la estructura lógica del texto? Además, se debe usar ejercicios de silogismos, analogías, secuencias numéricas, entre otras.

Justicia: Este estándar evalúa el pensamiento del estudiante en su capacidad de minimizar el favoritismo hacia su propia perspectiva y analizar todas las posturas con el mismo grado de rigurosidad. Aplicados al texto que se analiza, se propone la pregunta: ¿El autor consideró otros puntos de vista contrarios a los de él? Esto significa que el estudiante renuncia a estar de acuerdo con una postura y las pone a todas en tela de juicio, dejando a un lado los intereses particulares. Entonces se debe interrogar si el autor del texto hace esto con su propia postura y con las s contrarias.

Precisión: Los estudiantes deben detallar minuciosamente el problema, dar los rasgos más importantes. Por ende, se interroga por: ¿Qué detalles ofrece el autor sobre su punto de vista? ¿Hasta qué medida la información es precisa? Paul y Elder (2003) proponen las siguientes preguntas. “¿Puedes ser más específico?, ¿Puedes dar más detalles? y ¿Puedes ser más exacto?" (p. 9).

Profundidad: El estudiante debe ser capaz de juzgar hasta donde el texto aborda toda la complejidad del tema o si slo habla de este de forma superficial. Por ello es necesario indagar sobre: ¿En qué medida el autor habla del tema en toda su complejidad? ¿Considera el autor todos los aspectos importantes sobre el tema que está tratando? Paul y Elder (2003), plantean los interrogantes: "¿Qué factores hacen que el problema sea difícil?, ¿Cuáles son algunas de las complejidades de la pregunta? Y ¿Cuáles son algunas de las dificultades que tenemos que tratar?" (p. 9).

Amplitud: Es el estándar que revisa sobre otros puntos de vista contrarios a la lectura y si el autor los tuvo en cuenta. Se requiere dudar sobre: ¿Qué otras perspectivas contrarias al autor existen para abordar esta misma problemática y que sean válidas? Para Paul y Elder (2003) es necesario interrogar al educando sobre: "Necesitamos mirar esto 
desde otra perspectiva?, ¿Necesitamos considerar otro punto de vista? ¿Y necesitamos mirar esto de otra manera?" (p. 9).

\section{*Elementos del Pensamiento}

Para Elder (2005) los elementos del pensamiento son partes del pensamiento o elementos de razonamiento (p. 32) y permiten comprender la información que se desea validar, estos son:

Propósito: Todo pensamiento tiene una intención, toda lectura tiene un propósito. Por ende, es conveniente que se identifique la intención del autor.

Pregunta: Todo texto parte de una pregunta, el estudiante debe tener la capacidad para identificar la pregunta que está orientado el texto.

Supuestos: Son las creencias que damos por hecho, sin analizarlas, simplemente las aceptamos como verdaderas sin evidencia alguna.

Perspectiva: Cuando pensamos, lo hacemos desde una perspectiva. Además, es el punto de vista en donde se inscribe el autor.

Información: Son los datos, evidencias en que se basa el autor para expresar una idea.

Inferencia: Todo pensamiento contiene inferencias a partir de las cuales obtenemos conclusiones y damos significado a los datos y a las situaciones.

Concepto: Todo pensamiento se basa en ideas, juicios, conceptos, palabras. El estudiante debe analizar si el autor maneja los conceptos de forma clara o los distorsiona para manipular el pensamiento de los demás.

Implicaciones: Son las consecuencias que trae la información, es en lo que está inmersa una idea, hacia dónde nos dirige la información, qué podría pasar si la información se toma como válida.

\section{*Virtudes Intelectuales}

Según Paul y Elder (2005) las virtudes intelectuales no son naturales en la mente del ser humano y por tal hay que desarrollarlas. Son actitudes que distinguen al pensador crítico y buscan que el pensador no esté 
propenso al egocentrismo (creer que algo es cierto porque yo lo creo así) $o$ al sociocentrismo (creer que algo es cierto porque todos consideramos que es así), sino a usar la razón para enfrentar los problemas.

Humildad intelectual: Es reconocer los límites de nuestro conocimiento, para no pretender saber lo que no se sabe.

Entereza intelectual: Es examinarlo todo, hasta las propias creencias, sin aceptar nada como obvio.

Empatía intelectual: Es analizar el cómo la otra persona estructura su pensamiento y poder comprender el por qué llega a esa conclusión.

Autonomía intelectual: Es pensar por sí mismos, descubrir las cosas bajo su propia experiencia y dejar aún lado la confianza en la opinión de otros.

Integridad intelectual: Es valorar su propio juicio y el de los demás, con el mismo rigor. Aunque la mente busca aplicar mayor rigor a las posturas ajenas que a la propia.

Perseverancia intelectual: significa no rendirse ante las dificultades 0 complejidades, aunque la mente natural busca lo fácil y lo inmediato.

Confianza en la razón: Significa comprender las tendencias irracionales de la mente humana y trabajar para minimizarlas. Para ello, usan la razón y la evidencia para resolver los problemas, rechazando las creencias o supuestos.

Imparcialidad intelectual: cuando se piensa críticamente se juzga y evalúa todas las posturas con el mismo rigor, no muestra favoritismo alguno, ni con su propia tesis.

\section{B. Matthew Lipman}

La insistencia de Lipman (1992) es que una asignatura no se puede enseñar si no se enseña a pensar desde sí misma, es decir, no se debe enseñar filosofía, sino pensar filosóficamente. Agrega: "Si la educación tiene como meta lograr niños razonables, deben ser chicos que puedan al mismo tiempo pensar y reflexionar sobre las asignaturas de su instrucción" (p. 25). Por consiguiente, centra la enseñanza de la filosofía en el filosofar 
y no a un mero repetir de memoria el compendio de la historia de la filosofía (doxografía). Para ello, se basa en el ejemplo de Sócrates "que no enseña a saber filosofía sino a aplicarla" (p. 30). Esto lo conlleva a afirmar que la filosofía es la disciplina que mejor prepara al niño o al joven a pensar, lo cual debe ser el fin de toda educación: hacer sujetos razonables.

Además, las orientaciones que se pueden extraer de la lectura de Lipman (1992), "La Filosofía en el Aula", que permitan plantear textos filosóficos y pruebas de preguntas de selección múltiple basados en los Estándares Intelectuales y Elementos del Pensamiento aplicados a estudiantes de secundaria o primaria, serían:

Primero: Enseñar a pensar (a pensar mejor - pensamiento crítico), a pensar por sí mismo y no a memorizar (p. 103).

Segundo: El lenguaje debe estar contextualizado en el de los niños y jóvenes. Se debe traducir los textos al lenguaje propio de los pequeños: "lenguaje del aula" (p. 103) y no del profesorado, ni del adulto, ni tampoco del técnico filosófico (Claridad). Por eso Lipman propone que algunos deberían ser escritos por los mismos niños o los mismos jóvenes. Además, insiste que el estilo del texto sea narrativo, debido a la atracción que tienen estos textos con los niños y jóvenes.

Tercero: Ofrecer ejemplos de razonamiento e invitar a la discusión a través de preguntas y motive a que los estudiantes formulen sus propios interrogantes (p. 324) (Claridad).

Cuarto: Incentivar la rigurosidad del pensamiento, a valorar su propio pensamiento en criterios basados en la razón. Además, a revisar los criterios que usan para seleccionar dichos criterios (p. 295) (Certeza).

Quinto: Propiciar el pensamiento filosófico mediante la valoración de ideas, valorar argumentos lógicos, valorar sistemas conceptuales; además, debe desarrollar el manejo, el análisis y la construcción de conceptos (p. 114) (Lógica y concepto).

Sexto: Debe hacer que el estudiante reflexione sobre ¿Hacia dónde me puede conducir mis propias ideas? Que se le permita explorar los resultados de un razonamiento inválido (119) (Implicación). 
Séptimo: No debe inducir al estudiante a adoptar una postura específica que quiera el docente o el filósofo. Debe hacerle reflexionar críticamente todas las posturas, incluso razonar sobre sus propias creencias (Justicia), hasta las que contradiga al autor (Amplitud y perspectiva) (p. 146-147).

Octavo: Incentivar a reconocer la incoherencia en los argumentos del texto, de los compañeros y de los propios (p. 154) (Lógica). Junto con esto, debe ayudarles a desarrollar la capacidad de inferir, de concluir datos que no están presentes en el texto (p. 212-213) (Inferencia).

Noveno: Que les permita distinguir el propósito (Intención), en donde se requiere dar una justificación del porqué el autor dice lo que dice ( $p$. 244). Esto los debe hacer comprender sus propias intenciones, que es necesario especificar por qué yo hago las cosas.

Décimo: El estudiante deberá dar sentido y significado al texto que lee "descubriendo conexiones" (p. 147) en el mismo texto ¿En qué se basa el autor para decir esto? (Información) así tendrá más claro lo que se quiere decir.

Décimo primero: Descubrir nuevas alternativas, de pensar que la respuesta contraria a lo que él cree pueda ser válido (Amplitud). Debe ser capaz de tomar una postura de un autor y considerar una postura contraria -"encontrar su negación", en palabras de Lipman (1992, p 148-149).

La similitud entre Lipman y los autores de Paul y Elder, es que coinciden en ver al pensamiento crítico como el pensamiento que se autocorrige, que se mejora a sí mismo. Para esto Lipman plantea los planes de discusión y Paul con Elder, los estándares intelectuales y los elementos del pensamiento. Estas dos formas del pensamiento crítico, antes de ser contradictorias, se complementan, un claro ejemplo de ello se encuentra en Laurance Splitter y Ann Sharp (1996), en su texto "La otra educación (p. 84-85)" en donde plantean preguntas en los planes de discusión de las comunidades de indagación basadas en Estándares Intelectuales y en los Elementos del Pensamiento de la propuesta de Paul Richard.

\section{Peter Facione}

El pensamiento crítico, se debe centrar en el análisis de argumentos, del reconocimiento y evaluación de estos. Así el estudiante desarrollará 
habilidades de autoconfianza en la razón para tomar decisiones fundamentadas en el pensamiento crítico. Estas habilidades o disposiciones se concretizan en: interpretación, análisis, evaluación, inferencia, explicación y autorregulación (Facione, 2007, p. 4-7).

Además, la propuesta de Peter Facione se puede relacionar con la propuesta de Linda Elder y Paul Richard. Ver Cuadro de relación entre Facione y Paul de la tabla 1.

Tabla 1. Cuadro de relación entre Facione y Paul Richard

\begin{tabular}{|c|c|c|c|}
\hline Habilidad & Significado & $\begin{array}{l}\text { Interrogantes que lo } \\
\text { desarrollan }\end{array}$ & $\begin{array}{c}\text { Relación con la propuesta } \\
\text { de Elder y Paul }\end{array}$ \\
\hline \multirow{4}{*}{ Interpretación } & \multirow{4}{*}{$\begin{array}{l}\text { Comprender } \\
\text { y expresar el } \\
\text { significado o la } \\
\text { relevancia de } \\
\text { una gama de } \\
\text { experiencias, } \\
\text { hechos, datos, } \\
\text { creencias, ideas, } \\
\text { entre otras. }\end{array}$} & $\begin{array}{l}\text { ¿Puede usted pensar } \\
\quad \text { en ejemplos de } \\
\text { interpretación? ¿Puedes } \\
\text { parafrasear las palabras } \\
\text { de otro? }\end{array}$ & $\begin{array}{l}\text { Claridad: Puedes colocar } \\
\text { ejemplos de lo que estás } \\
\text { diciendo. Parafrasee el texto } \\
\text { anterior. }\end{array}$ \\
\hline & & $\begin{array}{l}\text { ¿Cómo le parece } \\
\text { reconocer un } \\
\text { problema y describirlo } \\
\text { imparcialmente? }\end{array}$ & $\begin{array}{l}\text { Justicia: Analizar los puntos } \\
\text { de vista de forma imparcial }\end{array}$ \\
\hline & & $\begin{array}{l}\text { ¿Sabes diferenciar la } \\
\text { idea principal de las } \\
\text { subordinadas? }\end{array}$ & $\begin{array}{c}\text { Relevancia: Identificar la } \\
\text { idea más relevante del } \\
\text { texto. }\end{array}$ \\
\hline & & $\begin{array}{l}\text { ¿Puedes identificar el } \\
\text { propósito, el tema o el } \\
\text { punto de vista del autor? }\end{array}$ & $\begin{array}{c}\text { Elemento de Propósito y } \\
\text { de Perspectiva. Reconocer } \\
\text { la intención del autor y su } \\
\text { punto de vista. }\end{array}$ \\
\hline \multirow{3}{*}{ Análisis } & \multirow{3}{*}{$\begin{array}{c}\text { Identificar las } \\
\text { relaciones de } \\
\text { inferencia entre } \\
\text { enunciados, } \\
\text { interrogantes y } \\
\text { demás, que tengan } \\
\text { la intención de } \\
\text { expresar algún juicio. }\end{array}$} & $\begin{array}{l}\text { ¿Puedes identificar } \\
\text { una suposición no } \\
\text { anunciada? }\end{array}$ & $\begin{array}{l}\text { Elemento de Suposición: } \\
\text { Evaluar los prejuicios o } \\
\text { ideas de que el autor da por } \\
\text { sentadas. }\end{array}$ \\
\hline & & $\begin{array}{l}\text { ¿Podrías escoger la } \\
\text { aseveración principal de } \\
\text { una editorial y rastrear } \\
\text { las diferentes razones } \\
\text { que presenta el editor } \\
\text { para apoyarla? }\end{array}$ & $\begin{array}{l}\text { Profundidad y el elemento } \\
\text { de información: Analizar los } \\
\text { datos presentes en un texto } \\
\text { para evaluar su profundidad. }\end{array}$ \\
\hline & & $\begin{array}{c}\text { ¿Puedes construir las } \\
\text { conclusiones principales } \\
\text { y los argumentos para } \\
\text { apoyarla? }\end{array}$ & $\begin{array}{l}\text { Lógica y el elemento de } \\
\text { inferencia: Analiza la } \\
\text { estructura de un texto y el } \\
\text { cómo llegar a inferir una } \\
\text { conclusión partiendo de los } \\
\text { argumentos de un texto. }\end{array}$ \\
\hline
\end{tabular}




\begin{tabular}{|c|c|c|c|}
\hline \multirow[t]{2}{*}{ Evaluación } & \multirow{2}{*}{$\begin{array}{l}\text { Es la valoración } \\
\text { de la credibilidad } \\
\text { de los enunciados } \\
\text { que describen } \\
\text { la percepción, } \\
\text { experiencia o juicio } \\
\text { de una persona. }\end{array}$} & $\begin{array}{l}\text { ¿Puedes juzgar } \\
\text { la credibilidad de } \\
\text { un autor con otras } \\
\text { interpretaciones } \\
\text { alternativas, } \\
\text { determinando así } \\
\text { la credibilidad de la } \\
\text { información? }\end{array}$ & $\begin{array}{c}\text { Certeza: Analizar } \\
\text { la credibilidad de la } \\
\text { información, validar la } \\
\text { fuente. } \\
\text { Amplitud: Analizar otras } \\
\text { posturas diferentes al del } \\
\text { autor. }\end{array}$ \\
\hline & & $\begin{array}{l}\text { ¿Puedes juzgar si la } \\
\text { conclusión sigue con } \\
\text { certeza de confianza a } \\
\text { sus premisas? }\end{array}$ & $\begin{array}{l}\text { Lógica y el elemento } \\
\text { de inferencia: Analiza } \\
\text { la relación y coherencia } \\
\text { entre las premisas con las } \\
\text { conclusiones. }\end{array}$ \\
\hline Inferencia & $\begin{array}{l}\text { Identificar y asegurar } \\
\text { los elementos } \\
\text { necesarios para } \\
\text { sacar conclusiones } \\
\text { razonables. }\end{array}$ & $\begin{array}{l}\text { ¿Puedes considerar la } \\
\text { información pertinente } \\
\text { de una premisa y sacar } \\
\text { las consecuencias que } \\
\text { se puedan rescatar para } \\
\text { llegar a una conclusión } \\
\text { lógica? }\end{array}$ & $\begin{array}{c}\text { Lógica y el elemento } \\
\text { de inferencia: Analiza } \\
\text { la relación y coherencia } \\
\text { entre las premisas con las } \\
\text { conclusiones. }\end{array}$ \\
\hline \multirow{2}{*}{ Explicación } & \multirow{2}{*}{$\begin{array}{l}\text { Es la capacidad } \\
\text { de presentar los } \\
\text { hallazgos del } \\
\text { razonamiento } \\
\text { personal de manera } \\
\text { reflexiva y coherente. }\end{array}$} & $\begin{array}{l}\text { ¿Puedes presentar } \\
\text { argumentos sólidos } \\
\text { y evidencias de tus } \\
\text { propias afirmaciones? }\end{array}$ & $\begin{array}{l}\text { Certeza: Confirmar si la } \\
\text { postura del autor es cierta o } \\
\text { falsa, basándose en criterios } \\
\text { lógicos y evidencias sólidas. }\end{array}$ \\
\hline & & $\begin{array}{l}\text { ¿Puedes enunciar la } \\
\text { evidencia que hallaste } \\
\text { para aceptar o rechazar } \\
\text { la postura del autor? }\end{array}$ & $\begin{array}{l}\text { Certeza: Confirmar si la } \\
\text { postura del autor es cierta o } \\
\text { falsa, basándose en criterios } \\
\text { lógicos y evidencias sólidas. }\end{array}$ \\
\hline \multirow{3}{*}{ Autorregulación } & \multirow{3}{*}{$\begin{array}{l}\text { Auto revisar o } \\
\text { controlar las } \\
\text { actividades y } \\
\text { resultados propios } \\
\text { de mi pensamiento } \\
\text { mediante el análisis } \\
\text { y la evaluación } \\
\text { para corregir el } \\
\text { pensamiento }\end{array}$} & $\begin{array}{l}\text { ¿Puedes revisar } \\
\text { diversos puntos de } \\
\text { vista en un tema } \\
\text { controversial, sin } \\
\text { basarme en mis propias } \\
\text { predisposiciones, } \\
\text { supuestos? }\end{array}$ & $\begin{array}{l}\text { Justicia y el elemento de } \\
\text { supuesto: Tiene en cuenta } \\
\text { diversos puntos de vista, } \\
\text { desarrollando la virtud } \\
\text { intelectual de Entereza } \\
\text { intelectual, de imparcialidad } \\
\text { e integridad intelectual. }\end{array}$ \\
\hline & & $\begin{array}{l}\text { ¿Puedes reconsiderar } \\
\text { su propia interpretación } \\
\text { o juicio en búsqueda } \\
\text { de un análisis más } \\
\text { profundo de los hechos } \\
\text { del caso? }\end{array}$ & $\begin{array}{c}\text { Profundidad y el elemento } \\
\text { de supuestos: Permite que } \\
\text { el estudiante auto valore } \\
\text { su pensamiento, evitando } \\
\text { sustentarse en supuestos } \\
\text { y profundizar de forma } \\
\text { persistente la complejidad } \\
\text { de un tema. }\end{array}$ \\
\hline & & $\begin{array}{l}\text { ¿Puedes cambiar su } \\
\text { opinión o conclusión al } \\
\text { darse cuenta de que sus } \\
\text { argumentos no son los } \\
\text { apropiados? }\end{array}$ & $\begin{array}{l}\text { Justicia y el elemento de } \\
\text { perspectiva: tiene en cuenta } \\
\text { de forma imparcial todos los } \\
\text { puntos de vista y reconoce } \\
\text { la humildad intelectual } \\
\text { cuando se equivoca. }\end{array}$ \\
\hline
\end{tabular}

Fuente. Facione (2007) y Paul (2005). Adecuación del autor. 


\section{Metodología}

Como diseño metodológico del proyecto, se retomó la teoría de la investigación cualitativa, que desarrolla Rodriguez, Gil, \& García (1999) "la cual la señalan como una investigación multimetódica en la que se busca interpretar, describir y comprender la realidad en su contexto natural, intentando interpretar los significados que tienen las personas que son objeto de estudio, lo que conlleva una gran recogida de datos para realizar los respectivos análisis" (p. 32). De forma complementaria Stake, citado por Rodriguez, Gil, \& García (1999), menciona que "el investigador cualitativo no descubre nada, sólo construye conocimiento en relación con la interpretación y comprensión de los significados que desarrollan las personas" (p. 34).

Por otro lado, el método fue Investigación-Acción (I-A.) tomando a Kemmis \& Mctaggart (1988) quienes la define como una forma de búsqueda autorreflexiva, llevado a cabo por participantes en situaciones de interacción social, como la educación, para comprender y mejorar las prácticas (p. 10).

Además, según Wilfred Carr (1988), expresa que "los objetos de la investigación-acción son sus propias prácticas educativas y su entendimiento de dichas prácticas, la cual debe mejorarlas" (p. 191). Por lo tanto, toda investigación acción empieza por un patrón de prácticas iniciales que, durante el proceso de mejoramiento, algunos elementos quedarán descartados, otros se mantendrán y otros serán añadidos, así se produce la transformación. Complementando la idea de mejoramiento de las prácticas educativas, estás se hacen posible en la medida que se aplique una espiral de ciclos en que la acción estará sometida a una programación monitoreada: planificación, acción, observación y reflexión... luego planificación, acción, observación y reflexión... (p. 197).

\section{Población y muestra}

Para la investigación realizada, se aplicó el proyecto de dos años, en el año 2017 y en el 2018, para la cual, se contó con una población de 95 estudiantes. Pero en la muestra del primer año, se aplicó a 37 estudiantes y en el 2018, sólo a 34 alumnos. 


\section{Técnicas de Recolección de Información}

Las técnicas de recogida de información nos permiten reducir de un modo sistemático la realidad social que deseamos estudiar, en este caso, la práctica pedagógica, a un sistema de representación que nos resulte fácil de tratar y analizar. Por ello es necesario responder "¿qué tipo de información busco? ¿Cómo quiero recogerla? ¿Cómo voy a registrarla?" (Latorre, 2005, p. 55).

Diario pedagógico: El diario pedagógico son registros que contienen información que ha observado el investigador o que ha hecho en vivo y contiene observaciones, reflexiones, interpretaciones y descripciones en el contexto natural.

Escalas de Medida: "Son instrumentos que se utilizan para determinar las disposiciones de los estudiantes hacia una acción manifiesta, ya sea a favor o en contra" (Latorre, 2005, p. 65). Las escalas se componen de un ítem y una serie de escalas a escoger, que puede ser "estimativas" (de acuerdo, poco de acuerdo, en desacuerdo) o "numérico" $(4,3,2,1$, donde uno es en la menor medida y cuatro en la mayor medida).

Pruebas: Es un instrumento de preguntas cerradas de selección múltiple y de preguntas abiertas que permite recoger información, a nivel cognitivo, sobre las habilidades de los estudiantes al seleccionar adecuadamente una opción. Dentro de las pruebas aplicadas, están las pruebas físicas y virtuales.

Cuestionarios de preguntas abiertas: "Los cuestionarios de preguntas abiertas se formulan para obtener respuestas expresadas en el propio lenguaje de la persona encuestada y sin un límite preciso de contestación" (Rodríguez, Gil, \& García 1999, p. 193).

Entrevistas: tienen el propósito de recoger información para comprender qué ocurre, qué se debe mejorar y conocer otros datos de la perspectiva del educando sobre el proyecto.

Por otro lado, "se aplica otra modalidad de entrevista llamada grupo de discusión o entrevista grupal" (Latorre, 2005, p. 75) y va dirigida a toda la muestra para conocer diversas perspectivas. 


\section{Validación de Instrumentos}

Para el proceso de validación, se realizó una aplicación diagnóstica de las pruebas, talleres, guías, entrevistas y cuestionarios en el año 2017 y que volvieron a ser aplicados en el año 2018 , dicho proceso consistió en: 1 . Se les preguntaba a los jóvenes sobre cada punto de las evaluaciones, sobre lo que entendía de las preguntas de los talleres, centrando la discusión en los criterios de comprensibilidad, ambigüedad, claridad y coherencia con los estándares intelectuales. 2. Se aplicaban las pruebas a un curso similar a la muestra, que hacía parte de la población y luego de ello, se les realizaba una entrevista grupal sobre las deficiencias de la prueba. 3. Desde la reflexión pedagógica del ciclo de la investigación-acción.

\section{Categorización}

La categorización está diseñada desde la propuesta de Linda Elder y Paul Richard del pensamiento crítico y la propuesta pedagógica, y por consiguiente se desarrolló el siguiente cuadro de las categorías de la tabla 2.

Tabla 2. Cuadro de categorías.

\begin{tabular}{|c|c|c|}
\hline Categoría & Sub-categoría & Indicadores \\
\hline \multirow{8}{*}{$\begin{array}{l}\text { Estándares } \\
\text { intelectuales }\end{array}$} & Claridad & Parafrasea, comprende conceptos \\
\hline & Certeza & $\begin{array}{l}\text { Verifica la información, valida la información, analiza } \\
\text { las fuentes }\end{array}$ \\
\hline & Relevancia & Identifica la idea principal, la intención del autor. \\
\hline & Lógica & Analiza la estructura del texto, identifica argumentos. \\
\hline & Justicia & Tiene en cuenta otros puntos de vista. \\
\hline & Precisión & Detalla información \\
\hline & Profundidad & Identifica la pregunta que originó al texto. \\
\hline & Ampliación & Plantea un punto de vista diferente. \\
\hline
\end{tabular}




\begin{tabular}{|c|c|c|}
\hline \multirow{8}{*}{$\begin{array}{l}\text { Elementos del } \\
\text { pensamiento }\end{array}$} & Propósito & $\begin{array}{l}\text { Identifica la intención del autor partiendo de } \\
\text { elementos explícitos e implícitos del texto. }\end{array}$ \\
\hline & Pregunta & $\begin{array}{c}\text { Analiza los posibles interrogantes que se planteó el } \\
\text { autor para escribir el texto. }\end{array}$ \\
\hline & Supuestos & $\begin{array}{l}\text { Valora los supuestos en que parte el autor para } \\
\text { fundamentar su punto de vista. }\end{array}$ \\
\hline & Perspectiva & $\begin{array}{l}\text { Identifica la postura del autor, su ideología y otras } \\
\text { perspectivas que contradicen al autor. }\end{array}$ \\
\hline & Información & $\begin{array}{l}\text { Determina cual es la información más útil para } \\
\text { responder las preguntas del taller. }\end{array}$ \\
\hline & Inferencia & $\begin{array}{l}\text { Identifica conclusiones, inferencias o } \\
\text { interpretaciones del autor frente a la información que } \\
\text { se maneja en la lectura. }\end{array}$ \\
\hline & Concepto & $\begin{array}{l}\text { Construye significados de las palabras claves, } \\
\text { partiendo del mismo contexto de la lectura. }\end{array}$ \\
\hline & Implicaciones & $\begin{array}{l}\text { Valora las consecuencias que trae el texto en el } \\
\text { contexto si es aceptado como válido. }\end{array}$ \\
\hline \multirow{8}{*}{$\begin{array}{c}\text { Virtudes } \\
\text { intelectuales }\end{array}$} & Humildad Intelectual & $\begin{array}{l}\text { Reconoce los límites de su propio conocimiento } \\
\text { durante las líneas de pensamiento. }\end{array}$ \\
\hline & Entereza Intelectual & $\begin{array}{c}\text { Analiza la validez del conocimiento, no acepta nada } \\
\text { como válido sin la revisión racional previa. }\end{array}$ \\
\hline & Empatía Intelectual & $\begin{array}{l}\text { Construye los puntos de vista y la lógica del } \\
\text { razonamiento de los compañeros a partir de lo que } \\
\text { expresan. }\end{array}$ \\
\hline & Autonomía Intelectual & $\begin{array}{c}\text { Analiza y cuestiona las cosas por sí mismo, sin } \\
\text { orientarse a respuestas inmediatas que dan los } \\
\text { demás. }\end{array}$ \\
\hline & Integridad Intelectual & $\begin{array}{l}\text { Somete su propio pensamiento al mismo rigor de } \\
\text { evidencia que le exige a los demás. }\end{array}$ \\
\hline & $\begin{array}{l}\text { Perseverancia } \\
\text { Intelectual }\end{array}$ & Analiza, sin renunciar, problemas complejos. \\
\hline & Confianza en la razón & $\begin{array}{c}\text { Escuchar los argumentos de los demás y resuelve } \\
\text { los problemas de forma inteligente. }\end{array}$ \\
\hline & Imparcialidad Intelectual & $\begin{array}{c}\text { Juzga todos los puntos de vista con los mismos } \\
\text { criterios, sin preferencia }\end{array}$ \\
\hline
\end{tabular}




\begin{tabular}{|c|c|c|}
\hline \multirow{5}{*}{$\begin{array}{l}\text { Propuesta } \\
\text { pedagógica }\end{array}$} & Dominio curricular & $\begin{array}{c}\text { La propuesta está ajustada a los estándares } \\
\text { intelectuales, elementos del pensamiento y virtudes } \\
\text { intelectuales para el desarrollo y mejoramiento del } \\
\text { pensamiento. }\end{array}$ \\
\hline & Planeación & $\begin{array}{l}\text { La propuesta cuenta con un plan organizado con } \\
\text { talleres, lecturas y recursos orientados al desarrollo } \\
\text { del pensamiento crítico. }\end{array}$ \\
\hline & Didáctica & $\begin{array}{c}\text { La didáctica implementada, usa metodologías y } \\
\text { diseña estrategias que mejora el pensamiento en los } \\
\text { estudiantes. }\end{array}$ \\
\hline & Evaluación & $\begin{array}{l}\text { Valora el desarrollo del pensamiento crítico y de las } \\
\text { habilidades cognitivas del estudiante a través de } \\
\text { pruebas abiertas y de selección múltiple. }\end{array}$ \\
\hline & Recursos & $\begin{array}{l}\text { Emplea los recursos virtuales necesarios para el } \\
\text { desarrollo de la propuesta pedagógica. }\end{array}$ \\
\hline
\end{tabular}

Fuente. Elaboración propia.

\section{Resultados}

Frente a los resultados hallados en el avance de los estándares intelectuales de los estudiantes de 11 A (2017-2018), se observa un histórico muy aleatorio en los niveles que arrojan las pruebas virtuales. Muy diferente a los resultados que arrojan los talleres que muestran una mejoría en la apropiación de los estudiantes al pensamiento crítico. De acuerdo con la definición de Paul Richard (2003) de que el estándar permite mejorar y evaluar el pensamiento, esta definición se halló en los estudiantes que comprendieron que el pensamiento crítico se relaciona con el mejorar la forma de pensar y que para ello se usaba la duda como estrategia principal. Por otro lado, desarrollar a cabalidad los estándares intelectuales requiere de mucho más tiempo, más de un año de intervención pedagógica y es necesario implementarlo desde el grado décimo.

Otro resultado significativo, se puede expresar en que los estudiantes ya no requieren del docente de forma continua para que les aclare o valide o les diga cuál es la respuesta correcta, sino que, desde el trabajo cooperativo, ellos argumentan y justifican cuál sería la respuesta más adecuada. (Ferreyra, 2014). 
Lo anterior reafirma su independencia que les permite fortalecer el estándar de certeza, porque buscan la verdad por sí mismos y no esperan que el docente les diga qué es lo que deben creer. Además, se mejoró el nivel del estándar de claridad y amplitud (Langer, 2016). Así mismo, los estudiantes mejoraron su comprensión lectora debido a que tienen más claridad en lo que leen, validan mejor la veracidad de la información, detallan elementos literales con precisión e identifican sin gran dificultad las posturas contrarias a las que argumentan las lecturas (de Almeida, Santos, Porto, 2016).

Sin embargo, continúa la dificultad al analizar a profundidad los textos, no valoran con rigurosidad las posturas del autor o las personales y se les dificulta identificar los datos más relevantes de una lectura.

En síntesis, en las pruebas aplicadas en los últimos ciclos, se muestra mejoría en el estándar de claridad, certeza y amplitud. Pero con serias dificultades en el de relevancia y precisión. Aunque en la última prueba de selección múltiple, mejoró el estándar de relevancia y hubo dificultades en justicia, precisión y lógica.

\section{Conclusiones}

Uno de los grandes obstáculos para el fomento de la actitud crítica en el estudiante es que no les gusta leer, desean que los datos se les transmitan por vías más sencillas con textos supremamente cortos. Esto requiere que, durante el mismo proceso de la intervención del docente, se motive a leer al educando. Para lo cual, las actividades no pueden caer en ser sólo lecturas sin un proceso de discusión, en dónde el joven pueda relacionar el problema con su vida real, comprender el tema desde su propia experiencia y pueda analizar sus propios razonamientos para mejorarlos (Konieczny, 2015).

Otra de las dificultades de la investigación, que limitaron el desarrollo del pensamiento crítico en su cabalidad, fue el no tener presente actividades que comprendieran secuencia gradual de actividades sencillas hacia complejas (Aparicio, 2018), en cada uno de los estándares y elementos del pensamiento.

Además, los talleres y actividades aplicadas no desarrollaron todos los estándares intelectuales como el de certeza, justicia y relevancia o 
elementos como información y perspectiva en los estudiantes (Tahull, 2016). Los estándares y elementos mencionados terminaron presentando dificultades, a pesar de que las estrategias y recursos usados. Por otro lado, se resalta que el estándar de claridad, de lógica y amplitud son los que presentan mejores avances en los alumnos; $y$, frente a los elementos del pensamiento, tenemos al concepto, inferencia y propósito, en los que se evidenciaron un mejor progreso. Por lo que conllevó a la reflexión del cómo promover el desarrollo de los otros cinco estándares y elementos del pensamiento que hacen falta por fortalecer.

Entonces, se llega a un análisis de estrategias que permitieran articular un estándar con un elemento y complementándose con una virtud intelectual. Lo que provocó también que se reestructura un nuevo orden gradual que partiera de un estándar sencillo al más complejo, buscando que se pueda desarrollar el pensamiento crítico en todos los Estándares Intelectuales de forma integral.

De acuerdo con lo anterior, se diseña una articulación, según la complejidad de los estándares intelectuales, elementos del pensamiento y virtudes intelectuales. Ver tabla 3.

Por último, como producto de la reflexión del mismo proceso investigativo, se obtuvo diversos productos que permiten orientar el desarrollo del pensamiento crítico, pero el más significativo fue el diseño de una rejilla se que ordenar las habilidades en los estudiantes en cuatro niveles (superior, alto, mínimo e inferior) y así orientar el fortalecimiento del pensamiento crítico en cualquier asignatura o docente que la desee aplicar. Ver tabla 4.

Tabla 3. Cuadro de articulación de Estándares, elementos y virtudes intelectuales.

\begin{tabular}{|c|c|c|c|}
\hline Orden & $\begin{array}{c}\text { Estándar } \\
\text { Intelectual }\end{array}$ & $\begin{array}{c}\text { Elemento del } \\
\text { pensamiento }\end{array}$ & Virtud intelectual \\
\hline 1 & Claridad & Concepto & Perseverancia Intelectual \\
\hline 2 & Relevancia & información & Autonomía Intelectual \\
\hline 3 & Lógica & Inferencia & Integridad Intelectual \\
\hline 4 & Precisión & Propósito & Confianza en la Razón \\
\hline 5 & Profundidad & Pregunta & Imparcialidad \\
\hline 6 & Justicia & Implicación & Empatía \\
\hline 7 & Amplitud & Perspectiva & Entereza \\
\hline 8 & Certeza & Supuesto & \\
\hline
\end{tabular}

Fuente. Adaptación de Paul Richard y Linda Elder (2005). 
Tabla 4. Cuadro de la Rejilla o rúbrica de los Estándares Intelectuales.

\begin{tabular}{|c|c|c|c|c|}
\hline $\begin{array}{l}\text { Estándar } \\
\text { Intelectual }\end{array}$ & Superior (A+) & Alto (A) & Mínimo (B) & Inferior (C) \\
\hline Claridad & $\begin{array}{c}\text { El estudiante } \\
\text { analiza la claridad } \\
\text { de las ideas } \\
\text { que expone } \\
\text { un autor en el } \\
\text { texto, parafrasea } \\
\text { correctamente } \\
\text { con ideas de } \\
\text { otros textos y } \\
\text { plantea nuevos } \\
\text { interrogantes. }\end{array}$ & $\begin{array}{c}\text { El estudiante } \\
\text { explica } \\
\text { claramente el } \\
\text { tema de un } \\
\text { texto; parafrasea } \\
\text { sin perder el } \\
\text { sentido de este, } \\
\text { planteando } \\
\text { preguntas claras y } \\
\text { precisas. }\end{array}$ & $\begin{array}{c}\text { El estudiante } \\
\text { explica el tema } \\
\text { de un texto de } \\
\text { forma regular. } \\
\text { Se le dificulta } \\
\text { dar ejemplos } \\
\text { y parafrasear } \\
\text { frases. }\end{array}$ & $\begin{array}{c}\text { Al estudiante se le } \\
\text { dificulta identificar } \\
\text { elementos } \\
\text { confusos o } \\
\text { complejos en un } \\
\text { texto; Se torna } \\
\text { incompetente } \\
\text { para explicar el } \\
\text { texto con sus } \\
\text { propias palabras. }\end{array}$ \\
\hline Certeza & $\begin{array}{c}\text { El estudiante, } \\
\text { da validez a } \\
\text { la información } \\
\text { presente en el } \\
\text { texto, siguiendo } \\
\text { criterios rigurosos. }\end{array}$ & $\begin{array}{l}\text { El estudiante } \\
\text { plantea criterios } \\
\text { rigurosos } \\
\text { para validar } \\
\text { la información } \\
\text { presente en el } \\
\text { texto. }\end{array}$ & $\begin{array}{l}\text { El estudiante } \\
\text { emite algunos } \\
\text { juicios sobre } \\
\text { su duda en la } \\
\text { veracidad de la } \\
\text { información. }\end{array}$ & $\begin{array}{c}\text { El estudiante se } \\
\text { muestra confuso } \\
\text { para comprobar } \\
\text { la veracidad de } \\
\text { la información } \\
\text { presente en el } \\
\text { texto. }\end{array}$ \\
\hline Relevancia & $\begin{array}{c}\text { El estudiante } \\
\text { analiza el nivel } \\
\text { de rigurosidad } \\
\text { en que el autor } \\
\text { maneja la } \\
\text { información para } \\
\text { abordar el tema } \\
\text { central del texto. }\end{array}$ & $\begin{array}{c}\text { El estudiante } \\
\text { determina la } \\
\text { información más } \\
\text { relevante del texto } \\
\text { en relación con el } \\
\text { tema del mismo. }\end{array}$ & $\begin{array}{c}\text { El estudiante } \\
\text { identifica algunas } \\
\text { ideas relevantes } \\
\text { del texto. }\end{array}$ & $\begin{array}{c}\text { El estudiante } \\
\text { presenta dificultad } \\
\text { para discriminar } \\
\text { la información } \\
\text { relevante con la } \\
\text { irrelevante en un } \\
\text { texto. }\end{array}$ \\
\hline Lógica & $\begin{array}{c}\text { El estudiante } \\
\text { ordena el texto en } \\
\text { su sentido lógico } \\
\text { e infiere con } \\
\text { exactitud el orden } \\
\text { de los párrafos } \\
\text { en su estructura } \\
\text { textual. }\end{array}$ & $\begin{array}{l}\text { El estudiante } \\
\text { comprende } \\
\text { con facilidad la } \\
\text { estructura del } \\
\text { texto. }\end{array}$ & $\begin{array}{l}\text { El estudiante } \\
\text { comprende } \\
\text { elementos } \\
\text { presentes en la } \\
\text { estructura del } \\
\text { texto. }\end{array}$ & $\begin{array}{c}\text { El estudiante } \\
\text { evidencia poca } \\
\text { habilidad para } \\
\text { identificar el } \\
\text { sentido lógico en } \\
\text { la estructura del } \\
\text { texto. }\end{array}$ \\
\hline Justicia & $\begin{array}{c}\text { El estudiante } \\
\text { analiza el nivel } \\
\text { de justicia (uso } \\
\text { de argumentos } \\
\text { sobre prejuicios) } \\
\text { del autor al tratar } \\
\text { otras posturas } \\
\text { contrarias. }\end{array}$ & $\begin{array}{l}\text { El estudiante } \\
\text { comprende la } \\
\text { forma como el } \\
\text { autor trata otras } \\
\text { posturas y la } \\
\text { manera como } \\
\text { favorece sus } \\
\text { ideas, usando } \\
\text { prejuicios o } \\
\text { distorsionando la } \\
\text { información. }\end{array}$ & $\begin{array}{c}\text { El estudiante } \\
\text { visualiza si la } \\
\text { lectura menciona } \\
\text { otras posturas } \\
\text { diferentes } \\
\text { a la suya; } \\
\text { identificando el } \\
\text { uso de prejuicios } \\
\text { o información } \\
\text { falsa. }\end{array}$ & $\begin{array}{c}\text { El estudiante } \\
\text { presenta dificultad } \\
\text { para analizar si el } \\
\text { autor considera } \\
\text { por igual } \\
\text { otras posturas } \\
\text { diferentes a la } \\
\text { suya. }\end{array}$ \\
\hline Precisión & $\begin{array}{c}\text { El estudiante } \\
\text { analiza si el autor } \\
\text { es preciso con la } \\
\text { información que } \\
\text { usa para abordar } \\
\text { el tema. }\end{array}$ & $\begin{array}{c}\text { El estudiante es } \\
\text { preciso con la } \\
\text { información del } \\
\text { texto }\end{array}$ & $\begin{array}{l}\text { El estudiante } \\
\text { aborda detalles } \\
\text { sobre el texto }\end{array}$ & $\begin{array}{l}\text { Al estudiante se } \\
\text { le dificulta dar } \\
\text { detalles sobre la } \\
\text { información del } \\
\text { texto o sobre el } \\
\text { pensamiento del } \\
\text { autor. }\end{array}$ \\
\hline
\end{tabular}




\begin{tabular}{|c|c|c|c|c|}
\hline Profundidad & $\begin{array}{l}\text { El estudiante } \\
\text { analiza si el autor } \\
\text { profundizó el } \\
\text { tema y ahondó } \\
\text { en temas más } \\
\text { complejos, no } \\
\text { quedándose en la } \\
\text { superficialidad. }\end{array}$ & $\begin{array}{l}\text { El estudiante } \\
\text { identifica los } \\
\text { aspectos más } \\
\text { complejos que } \\
\text { maneja el texto } \\
\text { sobre el tema } \\
\text { que se está } \\
\text { desarrollando. }\end{array}$ & $\begin{array}{c}\text { El estudiante } \\
\text { identifica algunos } \\
\text { elementos } \\
\text { complejos que } \\
\text { aborda el autor. }\end{array}$ & $\begin{array}{c}\text { El estudiante } \\
\text { solo identifica } \\
\text { elementos } \\
\text { superficiales que } \\
\text { aborda el autor. }\end{array}$ \\
\hline Amplitud & $\begin{array}{l}\text { El estudiante } \\
\text { identifica y } \\
\text { propone otras } \\
\text { perspectivas de } \\
\text { ver el problema } \\
\text { con un nivel } \\
\text { avanzado de } \\
\text { validez. }\end{array}$ & $\begin{array}{c}\text { El estudiante } \\
\text { identifica } \\
\text { diferentes puntos } \\
\text { de vista que son } \\
\text { diferentes al } \\
\text { autor, y con igual } \\
\quad \text { validez. }\end{array}$ & $\begin{array}{c}\text { El estudiante } \\
\text { identifica } \\
\text { algunas posturas } \\
\text { contrarias al autor } \\
\text { sin reconocer su } \\
\text { validez. }\end{array}$ & $\begin{array}{c}\text { A los estudiantes } \\
\text { se les dificulta en } \\
\text { identificar otra } \\
\text { postura contraria } \\
\text { al autor. }\end{array}$ \\
\hline
\end{tabular}

Fuente. Elaboración propia.

\section{Recomendaciones}

Como recomendación más pertinente del proyecto es orientar la construcción de preguntas de selección múltiple o preguntas abiertas, que promuevan el desarrollo de Estándares intelectuales y Elementos del Pensamiento. Para ello, se clasifica los niveles de dificultad en tres, desde lo literal, inferencial y crítico; permitiendo desarrollar de forma progresiva las habilidades de los educandos en torno al pensamiento crítico. Ver tablas 5 y 6 .

Tabla 5. Preguntas orientadoras para desarrollar los Estándares Intelectuales en las pruebas aplicadas.

\begin{tabular}{|c|c|c|c|}
\hline $\begin{array}{l}\text { Estándares } \\
\text { Intelectuales }\end{array}$ & Nivel III Crítico & Nivel II Inferencial & Nivel I Literal \\
\hline Claridad & $\begin{array}{l}\text { El autor ¿Enuncia } \\
\text { claramente su } \\
\text { intención? ¿El autor } \\
\text { conoce claramente el } \\
\text { tema en discusión? }\end{array}$ & $\begin{array}{l}\text { ¿Cómo se podría } \\
\text { ampliar este punto de } \\
\text { vista del autor? } \\
\text { Escriba ejemplos de lo } \\
\text { que dice el texto. }\end{array}$ & $\begin{array}{l}\text { ¿Cuál es el tema } \\
\text { central del texto? }\end{array}$ \\
\hline Certeza & $\begin{array}{l}\text { El autor ¿Demuestra } \\
\text { que lo que dice sea } \\
\text { verídico? ¿Qué } \\
\text { criterios puedo seguir } \\
\text { para probar esta } \\
\text { información? }\end{array}$ & $\begin{array}{l}\text { ¿Qué evidencias } \\
\text { pueden apoyar a lo que } \\
\text { se expresa en el texto? }\end{array}$ & $\begin{array}{l}\text { ¿Cómo evidencia } \\
\text { el autor lo que está } \\
\text { diciendo? }\end{array}$ \\
\hline Relevancia & $\begin{array}{c}\text { ¿Hasta qué medida } \\
\text { los datos son los más } \\
\text { pertinentes para el } \\
\text { tema tratado? ¿Qué } \\
\text { otros datos, olvida el } \\
\text { autor? }\end{array}$ & $\begin{array}{l}\text { El autor ¿Expresa } \\
\text { ideas relevantes al } \\
\text { tema en cuestión } \\
\text { o expresa datos } \\
\text { irrelevantes? }\end{array}$ & $\begin{array}{c}\text { ¿Qué datos centrales } \\
\text { hay en el texto sobre el } \\
\text { tema tratado? }\end{array}$ \\
\hline
\end{tabular}




\begin{tabular}{|c|c|c|c|}
\hline Lógica & $\begin{array}{c}\text { El texto ¿Es } \\
\text { internamente } \\
\text { consistente o contiene } \\
\text { contradicciones que no } \\
\text { explica? }\end{array}$ & $\begin{array}{l}\text { ¿Cómo se conecta } \\
\text { esta frase con el resto } \\
\text { del texto? ¿Cuál es la } \\
\text { estructura del texto? }\end{array}$ & $\begin{array}{l}\text { ¿Cuál es la idea } \\
\text { principal de cada } \\
\text { párrafo? }\end{array}$ \\
\hline Justicia & $\begin{array}{l}\text { ¿Muestra justicia el } \\
\text { autor o muestra sólo un } \\
\text { lado de la situación? }\end{array}$ & $\begin{array}{c}\text { ¿El autor usa } \\
\text { argumentos para } \\
\text { defender su postura de } \\
\text { otros puntos de vista o } \\
\text { tiene prioridad sobre el } \\
\text { suyo sin usar razones } \\
\text { lógicas? }\end{array}$ & $\begin{array}{l}\text { ¿El autor menciona } \\
\text { otros puntos de vista } \\
\text { contrarios a los de él? }\end{array}$ \\
\hline Precisión & $\begin{array}{c}\text { El autor ¿Da detalles } \\
\text { precisos sobre } \\
\text { el tema que está } \\
\text { desarrollando? }\end{array}$ & $\begin{array}{l}\text { ¿Qué otros detalles } \\
\text { están de forma } \\
\text { implícita en el texto? }\end{array}$ & $\begin{array}{l}\text { ¿Qué elementos del } \\
\text { texto son los más } \\
\text { precisos para el } \\
\text { problema tratado? }\end{array}$ \\
\hline Profundidad & $\begin{array}{l}\text { El autor ¿conduce al } \\
\text { lector en complejidades } \\
\text { importantes o enuncia } \\
\text { datos superficiales? }\end{array}$ & $\begin{array}{l}\text { La discusión que se } \\
\text { maneja en el texto ¿Se } \\
\text { cierra o queda abierta? }\end{array}$ & $\begin{array}{l}\text { ¿Hasta qué punto el } \\
\text { autor profundiza en el } \\
\text { tema? }\end{array}$ \\
\hline Amplitud & $\begin{array}{l}\text { El autor ¿considera } \\
\text { otros puntos de vista } \\
\text { relevantes o está } \\
\text { escribiendo con una } \\
\text { perspectiva muy corta? }\end{array}$ & $\begin{array}{l}\text { ¿Qué otros puntos de } \\
\text { vista diferentes del } \\
\text { texto existen? }\end{array}$ & $\begin{array}{l}\text { ¿Cuál es el punto de } \\
\text { vista del autor? }\end{array}$ \\
\hline
\end{tabular}

Fuente. Elaboración propia.

Tabla 6. Preguntas orientadoras para desarrollar los Elementos del Pensamiento en las pruebas aplicadas.

\begin{tabular}{|c|c|c|c|}
\hline $\begin{array}{l}\text { Elementos del } \\
\text { pensamiento }\end{array}$ & Nivel III Crítico & Nivel II Inferencial & Nivel I Literal \\
\hline Información & $\begin{array}{l}\text { ¿La información que } \\
\text { usa el autor es rigurosa } \\
\text { o parte de supuestos? }\end{array}$ & $\begin{array}{l}\text { ¿Qué usa el autor } \\
\text { para darle validez a su } \\
\text { texto? ¿Qué evidencias } \\
\text { presenta el autor de } \\
\text { sus afirmaciones? }\end{array}$ & $\begin{array}{c}\text { ¿De qué se trata el } \\
\text { texto? }\end{array}$ \\
\hline Concepto & $\begin{array}{c}\text { ¿Usa correctamente } \\
\text { el autor tal concepto } \\
\text { o lo distorsiona } \\
\text { para manipular el } \\
\text { pensamiento del } \\
\text { lector? }\end{array}$ & $\begin{array}{c}\text { ¿Con qué otros } \\
\text { conceptos se } \\
\text { relacionan el término? } \\
\text { ¿Cuál es el significado } \\
\text { de tal término, } \\
\text { siguiendo el contexto } \\
\text { de la lectura? }\end{array}$ & $\begin{array}{c}\text { ¿Cuál es el concepto } \\
\text { o conceptos claves del } \\
\text { texto? ¿Cuál es la idea } \\
\text { central? }\end{array}$ \\
\hline Pregunta & $\begin{array}{l}\text { ¿A qué pregunta busca } \\
\text { responder el autor con } \\
\text { el texto anterior? }\end{array}$ & $\begin{array}{l}\text { ¿Cuál es el sentido } \\
\text { de las preguntas } \\
\text { presentes en el texto? } \\
\text { ¿Para qué están esas } \\
\text { preguntas en el texto? }\end{array}$ & $\begin{array}{l}\text { ¿Cuáles son los } \\
\text { interrogantes que están } \\
\text { presentes en el texto? }\end{array}$ \\
\hline Inferencia & $\begin{array}{c}\text { ¿Qué conclusión se } \\
\text { puede inferir partiendo } \\
\text { de las premisas? } \\
\text { Siguiendo el orden } \\
\text { lógico del texto ¿Qué } \\
\text { párrafo debe seguir? }\end{array}$ & $\begin{array}{l}\text { ¿Cuál es el argumento } \\
\text { que defiende la tesis } \\
\text { central del autor? ¿Qué } \\
\text { tipo de argumento } \\
\text { usa el autor? ¿Cómo } \\
\text { llegó el autor a esa } \\
\text { conclusión? }\end{array}$ & $\begin{array}{c}\text { ¿Cuál es el argumento } \\
\text { que está presente en } \\
\text { el texto? }\end{array}$ \\
\hline
\end{tabular}




\begin{tabular}{|c|c|c|c|}
\hline Propósito & $\begin{array}{c}\text { ¿Cuál es la intención } \\
\text { del autor? }\end{array}$ & $\begin{array}{l}\text { ¿Cuál es la intención } \\
\text { comunicativa: Criticar, } \\
\text { exponer, argumentar, } \\
\text { informar, detallar...? }\end{array}$ & $\begin{array}{l}\text { ¿Cuál es el tipo de } \\
\text { texto de la lectura } \\
\text { anterior? }\end{array}$ \\
\hline Supuestos & $\begin{array}{l}\text { El autor del texto } \\
\text { ¿Cómo se aprovecha } \\
\text { de los supuestos del } \\
\text { lector para dar validez } \\
\text { a una información? }\end{array}$ & $\begin{array}{c}\text { ¿Qué supuestos están } \\
\text { de forma implícita en } \\
\text { la información? ¿En } \\
\text { qué creencias se } \\
\text { fundamenta el autor?, } \\
\text { ¿Qué suposición lleva } \\
\text { al autor a concluir esa } \\
\text { tesis? }\end{array}$ & $\begin{array}{l}\text { ¿Cuál es el supuesto } \\
\text { que está explícito en la } \\
\text { información? }\end{array}$ \\
\hline Perspectiva & $\begin{array}{c}\text { ¿Qué otras posturas } \\
\text { válidas hay diferentes } \\
\text { al texto? ¿Cómo se } \\
\text { refiere el autor a las } \\
\text { posturas contrarias } \\
\text { a él? }\end{array}$ & $\begin{array}{c}\text { ¿Qué postura maneja } \\
\text { el texto frente al } \\
\text { problema que se } \\
\text { plantea? }\end{array}$ & $\begin{array}{l}\text { ¿En qué está de } \\
\text { acuerdo y en qué está } \\
\text { contra el texto? }\end{array}$ \\
\hline Implicaciones & $\begin{array}{l}\text { ¿Qué implicaciones } \\
\text { o consecuencias } \\
\text { probables hay } \\
\text { si aceptamos la } \\
\text { información como } \\
\text { cierta? }\end{array}$ & $\begin{array}{c}\text { ¿Cómo presenta la } \\
\text { información el texto } \\
\text { para generar un } \\
\text { impacto en el lector? }\end{array}$ & $\begin{array}{c}\text { ¿Cuál es la idea que } \\
\text { quiere vender o hacer } \\
\text { convencer el autor con } \\
\text { el texto? }\end{array}$ \\
\hline
\end{tabular}

Fuente. Elaboración propia.

\section{Referencias bibliográficas}

Aparicio, O.Y. (2018). Las TIC como herramientas cognitivas para la investigación escolar. Revista Interamericana de Investigación, Educación y Pedagogía, RIIEP, 11(1). https://orcid.org/0000-0003-3535-6288

de Almeida, R. R., Santos, M. F., \& Porto, J. C. (2016). Lectura de textos ficcionales y el enfoque escolar de literatura: contribuciones para una Pedagogía de la Elección. Revista Interamericana de Educación, Pedagogía y Estudios Culturales, 9(1), 35-51. DOI: https://doi.org/10.22490/25391887.1925

de la Calle, C. V., Malaver, M. O., Gallego, J. D. M., Rodríguez, M., Flórez, J. C., Henao, C. E. \& Saldaña, R. (2014). Aportes de los doctorados de educación en ciencia, tecnología y sociedad, desde la sistematización de sus investigaciones doctorales científicas y formativas, 2000-2010. Revista Interamericana de Investigación, Educación y Pedagogía, RIIEP, 7(1). DOI: https://doi.org/10.15332/s1657-107X.2014.0001.04

Elder, R. L. (2005). Pensamiento Crítico para Niños. Recuperado de https://www. criticalthinking.org/resources/PDF/SP-Children_guide_all.pdf

Facione, P. (2007). Pensamiento Crítico: ¿Qué es y por qué es importante? Recuperado de http://www.eduteka.org/PensamientoCriticoFacione.php 
Fernández, A. (2006). Metodologías activas para la formación de competencias. Educatio siglo XXI (24), 35-56. Recuperado de http://revistas.um.es/educatio/article/ viewFile/152/135

Ferreyra, H. A. (2014). Mesas Socioeducativas para la Inclusión y la Igualdad. Un programa "De todos con todos". Una experiencia en construcción. Revista Interamericana de Investigación, Educación y Pedagogía, RIIEP, 7(2). DOI: https://doi. org/10.15332/s1657-107X.2014.0002.01

Kemmis, S., \& Mctaggart, R. (1988). Cómo planificar la Investigación Acción. Barcelona, España: Ediciones Laertes.

Konieczny, P. (2015). Lorenzo García Aretio: bases, mediaciones y futuro de la educación a distancia en la sociedad digital. Revista Interamericana de Investigación, Educación y Pedagogía, RIIEP, 8(1). DOI: https://doi.org/10.15332/s1657107X.2015.0001.08

Langer, E. (2016). La construcción de confianza para el estudio de prácticas de resistencia en la escolarización de jóvenes en contextos de pobreza urbana. Revista Interamericana de Investigación, Educación y Pedagogía, RIIEP, 9(2). DOI: https://doi. org/10.22490/25391887.1945

Lipman, M., Sharp, A. \& Oscanyan, F. (1992). Filosofía en el aula. Madrid, España: Ediciones la Torre.

Latorre, A. (2005). La Investigación Acción, conocer y cambiar la práctica educativa (3 ed.). Barcelona, España: Editorial Graó.

Paul, R. \& Elder L. (2003). Los fundamentos del Pensamiento Analítico. Recuperado de http://www.criticalthinking.org/resources/PDF/SP-Pensamientoanal\%C3\%ADtico.pdf

Paul, R. \& Elder L. (2005). Estándares de competencias para el pensamiento crítico. Recuperado de http://www.criticalthinking.org/resources/PDF/SP-Comp_Standards.pdf

Pérez, T. H. P. (2013). Aproximaciones al estado de la cuestión de la investigación en educación y derechos humanos. Revista Interamericana de Investigación, Educación y Pedagogía, RIIEP, 6(1). DOI: https://doi.org/10.15332/s1657-107X.2013.0001.05

Rodriguez, G., Gil, J., \& García, E. (1999). Metodología de la Investigación Cualitativa (2 ed.). Málaga: España. Ediciones Alije.

Splitter \& Sharp (1996). La otra educación. Buenos Aires: Argentina. Ediciones Manantial.

Tahull, J. (2016). Modernidad, educación y género. El proyecto inacabado. Revista Interamericana de Investigación, Educación y Pedagogía, RIIEP, 9(2), 159-178. DOI: https://doi.org/10.22490/25391887.1947 
Viejo, C. M., Cabezas, I. L., \& Martínez, M. D. J. I. (2013). Las redes de académicas en la docencia universitaria. Revista Interamericana de Investigación, Educación y Pedagogía, RIIEP, 6(2). DOI: https://doi.org/10.15332/s1657-107X.2013.0002.03

Wilfred, C., \& Stephen, K. (1988). Teoría Crítica de la Enseñanza. La Investigación Acción en la formación del profesorado. Barcelona: España. Ediciones Martinez Roa, S.A. 